\title{
EXPERIMENTAL AND NUMERICAL STUDY ON THE FLOW VISUALIZATION IN A TRI-HELICAL STATIC MIXER
}

\author{
Kuo-Tung Chang \\ Graduate Institute of Electro-Mechanical Engineering, Ming Chi University of Technology, Taishan, Taipei County, \\ Taiwan 243, R.O.C. \\ Jer-Huan Jang \\ Graduate Institute of Electro-Mechanical Engineering, Ming Chi University of Technology, Taishan, Taipei County, \\ Taiwan 243, R.O.C., jhjang@mail.mcut.edu.tw \\ Teng-Chuang Lai \\ Graduate Institute of Electro-Mechanical Engineering, Ming Chi University of Technology, Taishan, Taipei County, \\ Taiwan 243, R.O.C. \\ Jun-Nan Chen \\ Graduate Institute of Electro-Mechanical Engineering, Ming Chi University of Technology, Taishan, Taipei County, \\ Taiwan 243, R.O.C.
}

Follow this and additional works at: https://jmstt.ntou.edu.tw/journal

Part of the Engineering Commons

\section{Recommended Citation}

Chang, Kuo-Tung; Jang, Jer-Huan; Lai, Teng-Chuang; and Chen, Jun-Nan (2011) "EXPERIMENTAL AND NUMERICAL STUDY ON THE FLOW VISUALIZATION IN A TRI-HELICAL STATIC MIXER," Journal of Marine Science and Technology. Vol. 19: Iss. 4, Article 8.

DOI: $10.51400 / 2709-6998.2180$

Available at: https://jmstt.ntou.edu.tw/journal/vol19/iss4/8

This Research Article is brought to you for free and open access by Journal of Marine Science and Technology. It has been accepted for inclusion in Journal of Marine Science and Technology by an authorized editor of Journal of Marine Science and Technology. 


\title{
EXPERIMENTAL AND NUMERICAL STUDY ON THE FLOW VISUALIZATION IN A TRI-HELICAL STATIC MIXER
}

\author{
Kuo-Tung Chang*, Jer-Huan Jang*, Teng-Chuang Lai*, and Jun-Nan Chen*
}

Key words: flow visualization, tri-helical, static mixer.

\begin{abstract}
In this work, the flow visualization in a tri-helical static mixer has been investigated in detailed both experimentally and numerically. The static mixer used for the present study consists of four elements with a series of alternating left and right helical elements. Each element has the aspect ratio of 1.5 (i.e., $L / D=1.5$ ) and twisted angle of $180^{\circ}$. The objective of this investigation is mainly to explore the flow pattern inside a tri-helical static mixer due to its novelty and originality. It is clearly observed that the flows at the trailing edge for both numerical and experimental results are in a tri-lobe-like pattern. Apparently, the numerical solution matches with experimental result. It is also found that the rotation tri-lobe-like pattern flow decreases as the flow goes downstream due to viscous dissipation as well as the decrease of Reynolds number.
\end{abstract}

\section{INTRODUCTION}

Static mixers are simple and versatile pieces of equipment often used in continuous mixing [5], heat transfer [11], and chemical reactions applications $[3,7]$. They have good mixing characteristics, and since they have no moving parts, they can save capital and operating costs. Usually they are designed to disperse and mix two or more fluids in a short length of tube. Improved transport processes occurring in the static mixer such as flow division, radial eddying, flow constriction, and shear reversal eliminate the gradients in concentration, velocity and temperature. Static mixer is a device consisting of a series of flow orientation elements inserted along the axis of pipe. Pressure drives the fluid through the device, providing the energy needed to accomplish mixing. It has the advantage of being easy to install in existing systems. Pressure drop penalties caused by the destructive devices may be large.

Paper submitted 12/11/09; revised 03/08/10; accepted 04/18/10. Author for correspondence: Jer-Huan Jang (e-mail: jhjang@mail.mcut.edu.tw).

*Graduate Institute of Electro-Mechanical Engineering, Ming Chi University of Technology, Taishan, Taipei County, Taiwan 243, R.O.C.
However, fluid flow and mixing performance in theses device has not been rigorously characterized.

The scarcity of information about flow and mixing in static mixers is partially available due to their often-complex construction, which makes direct, non-intrusive experimental investigations difficult. The analytical solutions for velocity fields are also impractical due to the complex geometry of static mixers. Nevertheless, the static mixer has increased in popularity within the chemical industry over recent years. Because of a wide range of applications for static mixer, a variety of segment designs is available $[1,8,13]$. Despite widespread usage, the way these mixers work is still not fully understood. The design and optimization of mixers are traditionally performed by trial and error, with much depending on previous experience and wide safety margin. Computational fluid dynamics (CFD) is an increasingly effective alternative to speed up equipment design and gain additional fundamental understanding of mixing process. Hobbs and co-workers $[4,6]$ and Byrde and Sawley [2] numerically studied the helical static mixer for both the creeping flow and the laminar flow regimes (i.e., $10-5<\mathrm{NRe}<1000$ ). Rahmani et al. $[10,12]$ have also numerically investigated the flow pattern through a helical static mixer for both Newtonian and non-Newtonian fluids, and provided useful information that can be extracted from the simulation results.

Recently, Lin et al. [9] developed a novel invention named as tri-helical static mixer. The static mixer consists of four elements. The mixing elements, which are called segments, as one could imagine, appear to have been cut from a long periodic structure. Each segment has three helical channels and is twisted through an angle of $180^{\circ}$. These segments are inserted in-line and housed in a pipe that squeezes the liquid through the resulting mixing elements. The tri-helical static mixer consists of left- and right-twisting helical elements at right angles to each other. The complete mixer consists of a series of elements of alternating clockwise and counterclockwise twist arranged axially within a pipe so that the leading edge of an element is at $60^{\circ}$ angles to the trailing edge of the previous element. Fig. 1 shows the configuration and dimension of this element. Mixing in the elements occurs through a combination of flow splitting and shearing at the junctions of successive elements and a stretching and folding mechanism 


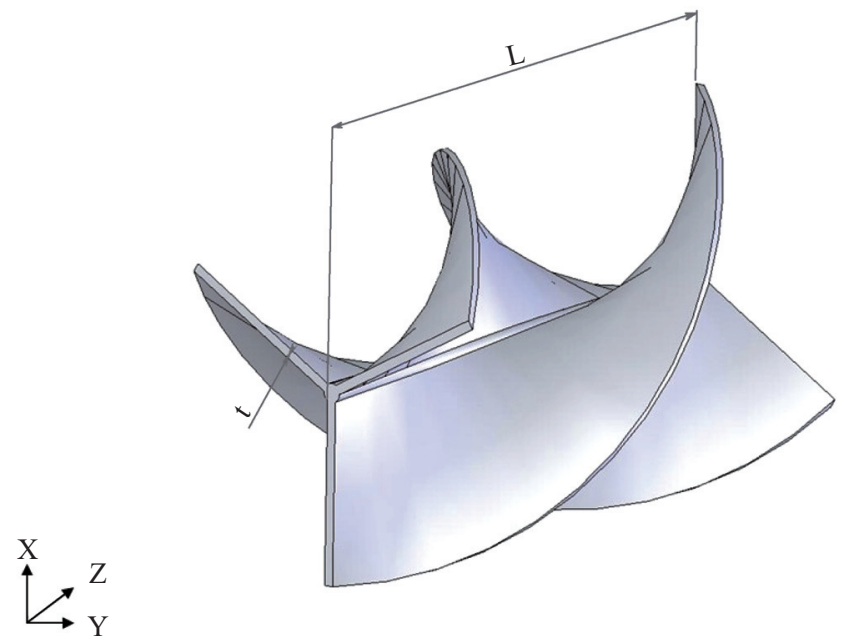

Fig. 1. Sketch of tri-helical static mixer elements.

within the elements. This makes the tri-helical element as an excellent radial mixing device.

From the literatures cited above, it is found that experimental work for static mixers has never been under investigation. A novel design of tri-helical static mixer has been developed for better mixing processes. However, the flow characteristics of tri-helical static mixers have never been discovered. This motivates the present study. The main purpose of present study is to explore the flow pattern inside a tri-helical static mixer due to its novelty and originality. The mixing efficiency of the tri-helical static mixer will be investigated in the future.

\section{EEPERIMENTAL SETUP AND PROCEDURES}

\section{Experimental Setup}

The objective of this investigation is to observe the flow field within a tri-helical static mixer numerically and experimentally. The fluid utilized in the study is water, and the experiment was carried out within a water channel. Experimental setup includes a Nd-YAG laser as the laser sheet source, a tri-helical static mixer as testing module, upper and lower water tanks, seeders and imaging capture system. The image capture system includes a digital camera, a CCD camera, and a desktop computer. The experimental setup is shown in Fig. 2. It should be noted that a filter with honeycomb is placed before the channel in order to obtain a uniform flow.

\section{Testing Module}

In this paper, a tri-helical static mixer was utilized by inserting into a tube for flow visualization. The tri-helical static mixer divides the pipeline into three parts. The testing modules consist of four elements with a series of alternative left and right helical elements arranged axially within a pipe so that the leading edge of an element is at $60^{\circ}$ angles to the trailing edge of the previous element. The dimension of each

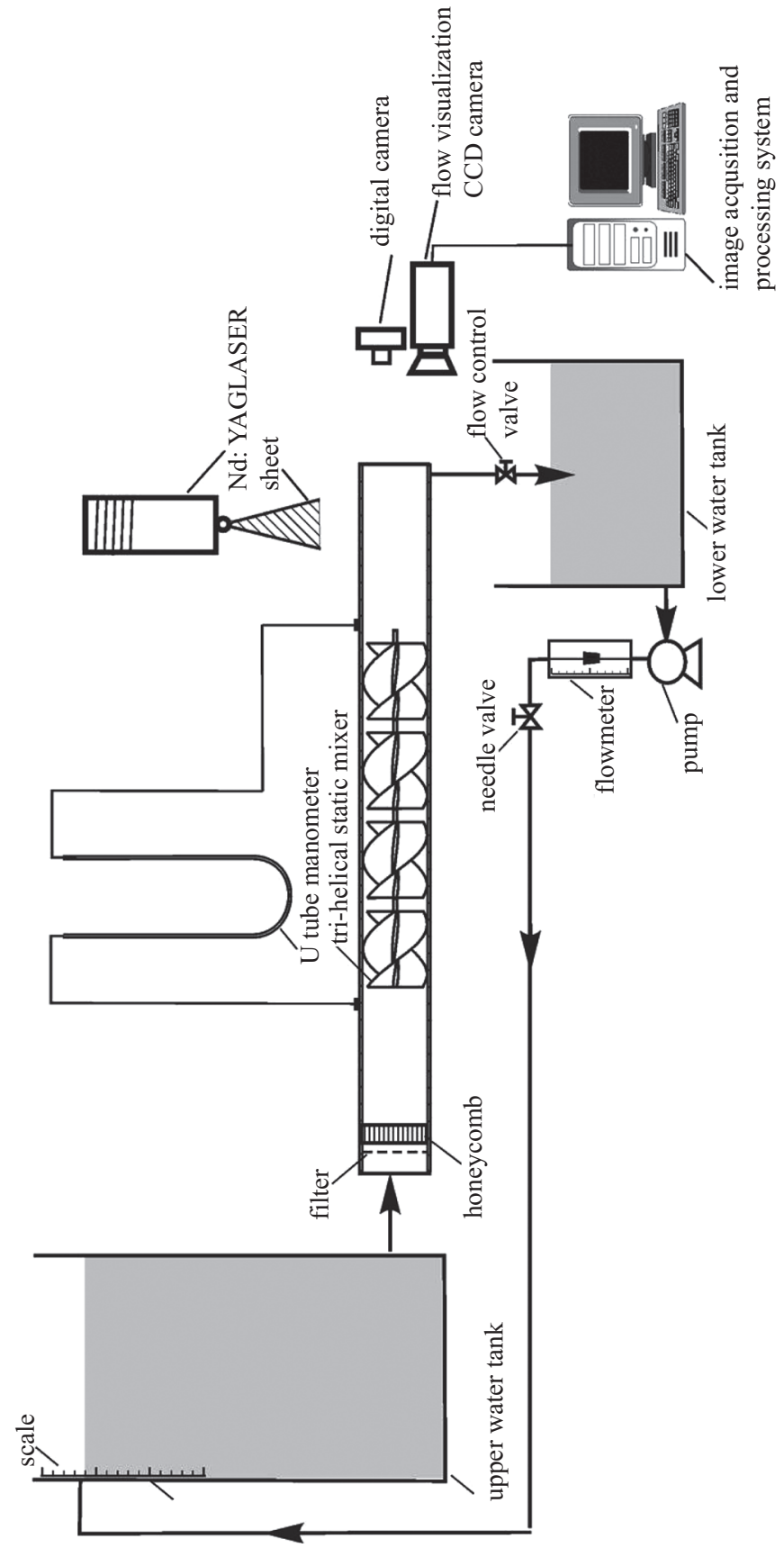

Fig. 2. Illustration of experimental setup.

element is $40 \mathrm{~mm} \times 60 \mathrm{~mm}$. So the aspect ratio for each element is 1.5 . The rib thickness is $1.2 \mathrm{~mm}$. The sketch of the right helical element is presented in Fig. 1. The diameter of the pipe is $40.4 \mathrm{~mm}$ such that there is a small gap between the tri-helical static mixer and the pipe. The total length of the pipe is $1000 \mathrm{~mm}$, while the length of the testing module is 240 $\mathrm{mm}$. This arrangement is shown in Fig. 3.

\section{Visualization}

The main equipment of visualization is the Nd-YAG laser sheet source. It is a portable sized industrial laser, which 


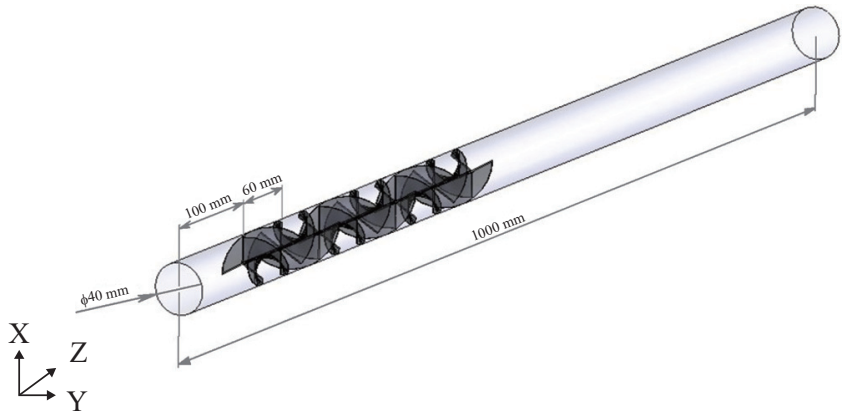

Fig. 3. The placement and configuration of the tri-helical static mixer in the water channel.

is solid state type excited by a semi-conductor diode. There is a cylindrical optics in front of the laser head to generate a laser sheet. The expansion angle of the laser sheet is $20^{\circ}$, and the thickness of the laser sheet is $0.5 \sim 1 \mathrm{~mm}$. Its color is green with wavelength of $532 \mathrm{~nm}$, and the maximum power is 200 $\mathrm{mW}$ with the input voltage of $110 \mathrm{~V}$ AC. Plastic particles are made of polyamide and seeded into water tank to scatter the laser light. The diameters of the particles are between $30-70$ $\mu \mathrm{m}$ and the specific weight is 1.03 at $25^{\circ} \mathrm{C}$. Because the particles are small enough as that they do not diffuse from the flow. Since the specific weight of the plastic powder is greater than that of water, an observation was made to verify the effect of density difference between ABS particle and water. It is found that the seeders would remain still in the water without any disturbance, and it took a day for seeders to fall off on the bottom of the tank. Therefore, it is adequate for this experiment to use the seeders. It should be noted that there has to be enough concentration of the seeders in order to obtain enough tracking lines for the analysis.

\section{Experimental Procedure}

After seeders are planted into the water, the pump was started for experiment. The water flow is controlled by the needle valve. After the flow reaches steady condition and the $\mathrm{Nd}-Y A G$ laser is set at a desired location, the image acquisition system records the flow image for both digital picture and digital video. The acquisition frequency is $60 \mathrm{~Hz}$ and the resolution is $640 \times 480$ pixels for digital video. The capture speed for digital picture is set at $1 / 4 \mathrm{~s}-1$ with the aperture of 8 . The resolution for digital picture is $2048 \times 1536$ pixels.

\section{NUMERICAL COMPUTATION}

In the present study, the flow is considered to be three dimensional, laminar, steady and incompressible. The properties of the working fluid (water) are also assumed to be constant. The governing equations contain continuity equation and the momentum equations in three directions. The solution to the governing equations is performed by using finite-volume method of upwind difference scheme. Both continuity equation and momentum equations are solved with solid boundary

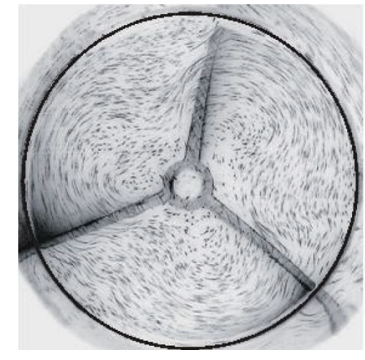

(a)

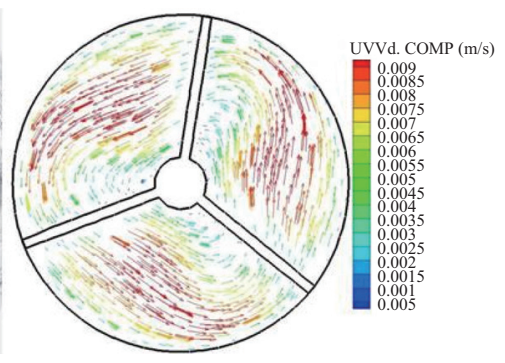

(b)
Fig. 4. (a) Projected velocity vectors at the trailing edge of Tri-helical static mixer; (b) particle trajectory at the trailing edge of Tri-helical static mixer.

of no-slip condition. Entrance condition is assigned the entering velocity which is obtained with required Reynolds number. The definition of Reynolds number is expressed as the following:

$$
\operatorname{Re}=\frac{\rho V_{a} D}{\mu}
$$

where $\rho$ is the density, $V_{a}$ is the averaged velocity, $D$ is the inner diameter of the tube, and $\mu$ is the viscosity.

Exit condition is set as outlet of the flow. The model domain of present study is divided into a number of cells as control volumes. In the finite volume method, the governing equations are numerically integrated over each of these computational cells or control volumes. The simulations were performed by using commercial software of STAR-CD with version 3.24. The convergence criteria are set as the residual is less than $10^{-5}$. Pro-surf (grid generator) is used to generate grid on the surfaces of tri-helical static mixer. There are total of 50200 grids in the computation domain.

\section{MODEL VALIDATION}

Validation of the 3D numerical model is carried out by comparing numerical result with experimental works. To the author's knowledge, this paper is the first study of static mixers to visualize the flow pattern at the downstream of a tri-helical static mixer. Fig. 4 numerically and experimentally shows the flow pattern of the tri-helical static mixer at the trailing edge for $\mathrm{Re}=1500$. It is clearly observed that the flows for both numerical and experimental results are in a tri-lobe-like pattern. Apparently, the numerical solution is quite comparable to that of experimental work. This confirms that the present numerical model is capable to describe the flow within the tri-helical static mixer in detail.

The cross-sectional flow at the trailing edge for numerical result also shows in counterclockwise direction, however, that of experimental result is not able to be found with present facility. Nevertheless, the flow at the downstream of the trihelical static mixer can be recorded from a video and shown in 


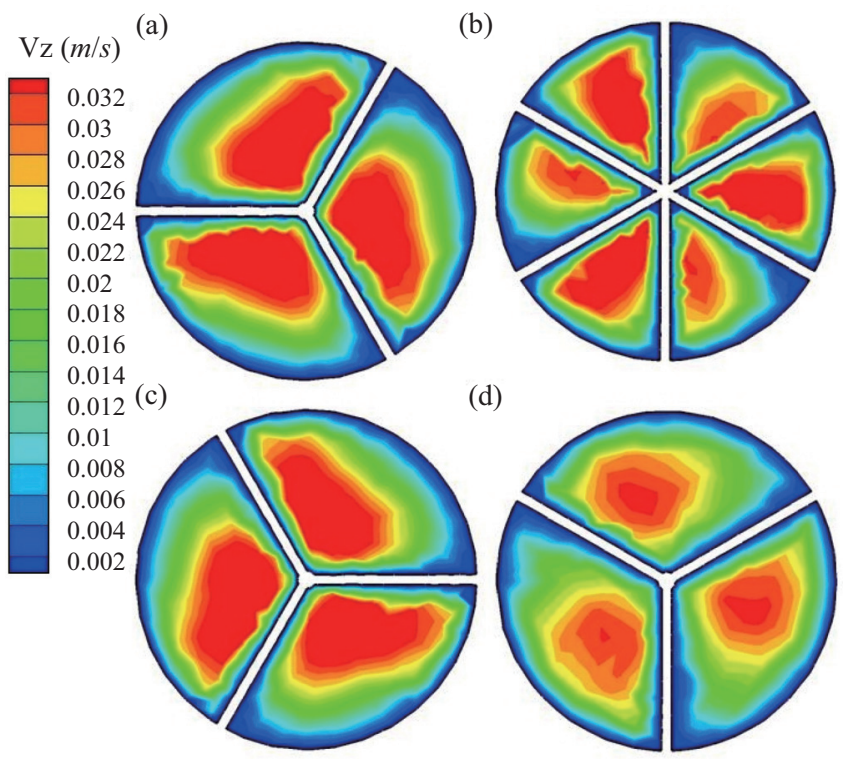

Fig. 5. Axial velocity distribution at various locations; (a) middle of third element; (b) at the intersection of the third and fourth mixing elements; (c) in the middle of the fourth mixing element; and (d) at the trailing edge of the fourth mixing element.

counterclockwise direction. It is also observed in Fig. 4(a) that the cross-sectional projected velocity of the flow reaches its maximum after passing the element rib. In Fig. 4(b), the trajectory becomes longer at the corresponding area of larger cross-sectional projected velocity. This indicates again that the simulation results have close comparison with experimental ones.

\section{RESULTS AND DISCUSSION}

For the present study, numerical calculations are performed at three Reynolds numbers, 800, 1500, and 2000. The axial velocity, radial velocity, and vorticity of the fluid are obtained and presented for four different axial locations. Figs. 5, 6, and 7 depict the cross-sectional distribution of axial velocity, radial velocity, and vorticity, respectively. These properties are presented through the computation domain at four different locations along the axial direction. The four locations are: (i) in the middle of the third mixing element; (ii) at the intersection of the third and fourth mixing elements; (iii) in the middle of the fourth mixing element; and (iv) at the trailing edge of the fourth mixing element.

Fig. 5(a) shows the flow was split into three regions by the static mixer. It is seen that larger axial velocities can be found on the right of each flow channel and close to the center region of the pipe. This is because the third element is twisted clockwise. As further downstream at the intersection of the third and fourth elements as shown in Fig. 5(b), the flow in one channel is separated into two parts. The high axial velocity is mixing with the low axial velocity. Clearly, the velocity difference generates shear strain and in turn

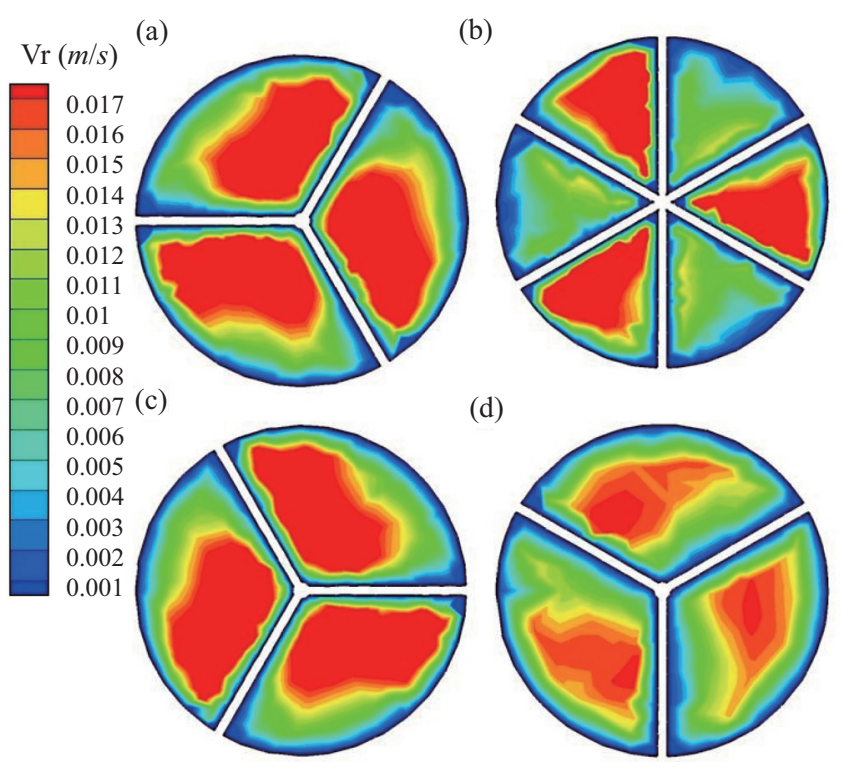

Fig. 6. Radial velocity distribution at various locations; (a) middle of third element; (b) at the intersection of the third and fourth mixing elements; (c) in the middle of the fourth mixing element; and (d) at the trailing edge of the fourth mixing element.

more mixing is resulted. As the flow goes further downstream in Fig. 5(c), the axial velocity distribution displays a similar pattern as Fig. 4(a), while larger axial velocities are observed on the left of each flow channel. This is also due to the twisting counterclockwise direction of the fourth element. As the flow comes to the trailing edge shown in Fig. 5(d), the peak value of axial velocity is located at the center for each channel. However, the peak value becomes smaller than that for the upstream.

The cross-sectional distributions of radial velocity at four locations are presented in Fig. 6. It is clearly observed in Fig. 6(a) that the radial velocities are large from the center to the wall of the pipe and appear on the right of each channel. This indicates the flow is forced towards to the wall. As the flow comes to the intersection of the third and fourth element in Fig. 6(b), the radial velocity is small for the fourth element, but larger radial velocity remains in the third element. This indicates a transverse velocity change in the flow channel. The radial velocities in the fourth element shown in Fig. 6(c) present a similar pattern as that in Fig. 6(a). However, the larger radial velocities appear on the left of each channel. Fig. 6(d) shows the radial velocity decreases at the trailing edge of the fourth element. It can be concluded by the decrease of both axial and radial velocities that the transverse velocity increases at the trailing edge of the fourth element.

The mean axial vorticity distributions for four axial locations are presented in Fig. 7. The mean axial votitcity is calculated from the velocity field as

$$
\Omega_{Z}=\frac{\partial V}{\partial X}-\frac{\partial U}{\partial Y}
$$




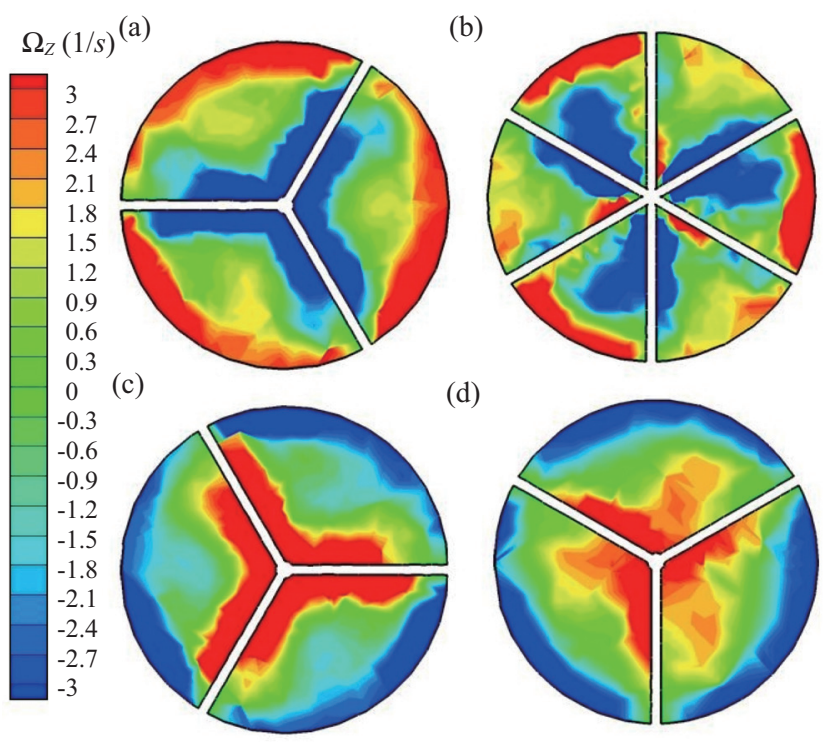

Fig. 7. Vorticity distribution at various locations; (a) middle of third element; (b) at the intersection of the third and fourth mixing elements; (c) in the middle of the fourth mixing element; and (d) at the trailing edge of the fourth mixing element.

and is a measure of the strength of the vortex flow. Positive vorticity represents counterclockwise rotation, while negative value indicates clockwise rotation. It is noted that the axial vorticity is high close to the pipe wall and close to the rib in Fig. 7(a). However, the rotation direction at the wall is positive and negative at the rib. As further downstream at the interface of the third and fourth element in Fig. 7(b), the positive axial vorticity close to the wall for the fourth element decreases and is produced at the center region. Also, the negative axial vorticity starts to migrate towards to the wall. As the flow goes further downstream shown in Fig. 7(c), the positive axial vorticity is located at the center of the pipe, while the negative axial vorticity is located at the pipe wall. This value is opposite to that in Fig. 7(a), which implies the vorticity rotates opposite direction. This is due to the opposite twisted direction of the third and fourth elements. It is clear in Fig. 7(d) that the vorticity is decaying at the downstream of the mixer with peak value at the center.

Fig. 8 presents the axial velocity distribution along the flow direction with and without a tri-helical static mixer. The axial velocity for flow without a static mixer is a standard developing flow distribution and the axial velocity at the center of the pipe doubles at the exit of the pipe. It is noticed that the axial velocity is higher at the entrance region for the flow with a tri-helical static mixer. Obviously, the increase in axial velocity is because of the back pressure by the placement of static mixer. Clearly, the axial velocity becomes larger in the separated channels inside the static mixer. The flow is affected by the helical channel, and the fluid at the center is forced to move to pipe wall. Since the consecutive elements are in an alternative left and right helical arrange ment. It should be

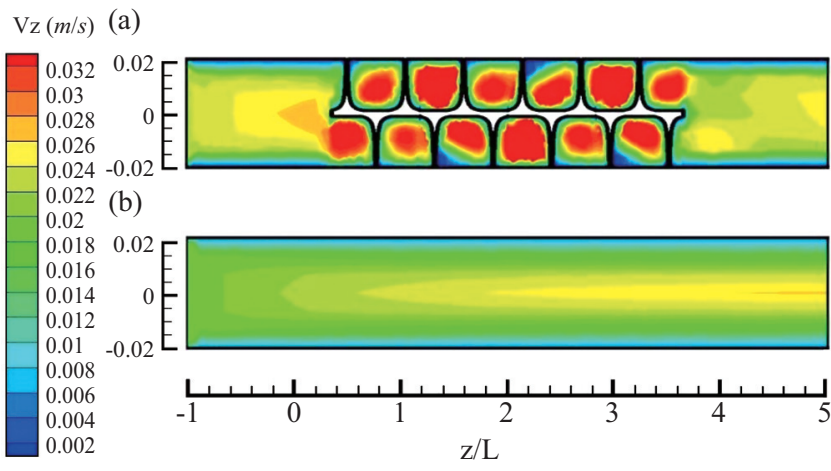

Fig. 8. Axial velocity distribution along flow direction: (a) with and (b) without a tri-helical static mixer.

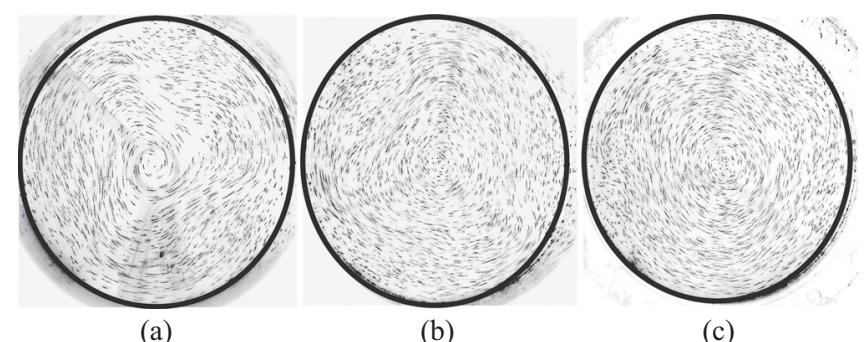

Fig. 9. The cross-sectional flow pattern at three location for $\operatorname{Re}=1500$; (a) trailing edge of the mixer; (b) 1D after the trailing edge; (c) 2D after the trailing edge.

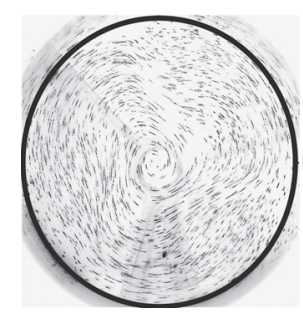

(a)

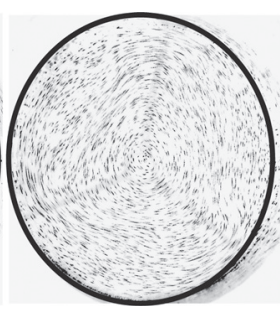

(b)

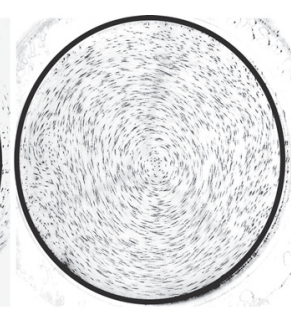

(c)
Fig. 10. The cross-sectional flow pattern at the trailing edge of the mixer for (a) $\operatorname{Re}=800$; (b) $\operatorname{Re}=1500$; (c) $\operatorname{Re}=2000$.

noted that there is not stagnation region inside the static mixers, but the velocity change is severe. In turns, increase of mixing performance is obtained.

The cross-sectional flow pattern at three location for $\mathrm{Re}=$ 1500 is presented in Fig. 9. The three locations are the exit at the trailing edge, 1D from the trailing edge, and 2D from the trailing edge, respectively. Comparing the figures, it is noted that the lobe-like shape becomes not so apparent for further downstream. This can be explained by the viscosity of the fluid dissipating the flow energy and smoothing out the flow pattern. This can be concluded as the effect of tri-helical static mixer becomes insignificant at the downstream .It is also found that the trajectories close to the pipe wall is shorter than those close to the center. This indicates that the velocities close to the center is larger than those close to the pipe wall. 
This pattern is similar to a rotational motion in a pipe. However, the flow at the center rotates and becomes tri-lobe-like pattern due to tri-helical static mixer.

Fig. 10 shows the flow field at the trailing edge of the exit experimentally with three Reynolds numbers, $\mathrm{Re}=800,1500$, and 2000. Obviously, the trajectories are longer for higher Reynolds numbers. This means that the overall cross-sectional averaged velocity is higher for higher Reynolds numbers. It is also noticed that the tri-lobe-like pattern becomes less significant as Reynolds number decreases. This is due to the fact that the viscous effect is less for higher Reynolds number. Hence the distance for mixing process after mixing element would be longer as Reynolds number increases.

\section{CONCLUSIONS}

The flow visualization in a tri-helical static mixer has been studied in detailed numerically and experimentally. To the authors' knowledge, this is the first experimental study of flow visualization for a tri-helical static mixer. The CFD model using STAR-CD was successfully developed to predict a laminar flow in a tri-helical static mixer. Results show the numerical simulation has comparably match with that of experimental work. Both numerical and experimental results have been observed that the flow pattern at the trailing edge of the exit is in a tri-lobe-like pattern. In addition, the dissipation of mixing flow at the downstream increases as Reynolds number decreases as well as exit distance increases.

\section{NOMENCLATURES}

$D \quad$ inner diameter of the pipe

$L \quad$ length of static mixer element

$U, V, W \quad$ velocity component in $X, Y, Z$ direction

$V_{a} \quad$ averaged velocity

$V_{\mathrm{r}} \quad$ radial velocity

$V_{\mathrm{z}} \quad$ axial velocity
$X, Y, Z$
coordinate direction
$\Omega_{\mathrm{Z}}$ axial vorticity

\section{REFERENCES}

1. Bakker, A., LaRoche, R. D., and Marhsall, E. M., Laminar Flow in Static Mixers with Helical Elements, The Online CFM book at http://www. bakker.org (2000).

2. Byrde, O. and Sawley, M. L., "Optimization of a Kenics static mixer for non-creeping flow conditions," Chemical Engineering Journal, Vol. 72, pp. 163-169 (1999).

3. Chen, R. C., "Analysis of homogeneous slurry pipe flow," Journal of Marine Science and Technology, Vol. 2, pp. 37-45 (1994).

4. Hobbs, D. M. and Muzzio, F. J., "Reynolds number effects on laminar mixing in the Kenics static mixer," Chemical Engineering Journal, Vol. 70, pp. 93-104 (1998).

5. Hobbs, D. M. and Muzzio, F. J., "Optimization of a static mixer using dynamical systems techniques," Chemical Engineering Science, Vol. 53, No. 18, pp. 3199-3213 (1998).

6. Hobbs, D. M., Swanson, P. D., and Muzzio, F. J., "Numerical characterization of low Reynolds number flow in the Kenics static mixer," Chemical Engineering Science, Vol. 53, No. 8, pp. 1565-1584 (1998).

7. Jones, S. C., Sotiropoulos, F., and Amirtharajah, A., "Numerical modeling of helical static mixers for water treatment," Journal of Environmental Engineering, Vol. 128, No. 5, pp. 431 - 440 (2002).

8. Kumar, V., Shrike, V., and Nigam, K. D. P., "Performance of Kenics static mixer over a wide range of Reynolds number," Chemical Engineering Journal, Vol. 139, pp. 284-295 (2008).

9. Lin, C. T., Chen, K. D., and Chang, K. D., "Static mixer along with its helical mixing device," PRC Patent No. ZL 200720126548.

10. Rahmani, R. K., Keith, T. G., and Ayasoufi, A., "Three-dimensional numerical simulation and performance study of an industrial helical static mixer," Journal of Fluids Engineering, Vol. 127, pp. 467-483 (2005).

11. Rahmani, R. K., Keith, T. G., and Ayasoufi, A., "Numerical study of the heat transfer rate in a helical static mixer," ASME Journal of Heat Transfer, Vol. 128, pp. 769-783 ( 2006).

12. Rahmani, R. K., Keith, T. G., and Ayasoufi, A., "Numerical simulation and mixing study of pseudoplastic fluids in an industrial helical static mixer," Journal of Fluids Engineering, Vol. 128, pp. 467-480 (2006).

13. Thakur, R. K., Vial, Ch., Nigam, K. D. P., Nauman, E. B., and Djelveh, G., "Static mixers in the process industries-a review," Chemical Engineering Research and Design, Vol. 81, No. 7, pp. 787-826 (2003). 